\title{
Simultaneous Pellian equations with a single or no solution
}

by

\author{
Alain Togbé (Westville, IN) and Bo He (Neijiang)
}

1. Introduction. A system of simultaneous Pellian equations is a system of Diophantine equations of the form

$$
a x^{2}-b y^{2}=\delta_{1}, \quad c y^{2}-d z^{2}=\delta_{2},
$$

where $a, b, c, d, \delta_{1}, \delta_{2}$ are nonzero integers, and $\operatorname{gcd}\left(a b, \delta_{1}\right)=\operatorname{gcd}\left(c d, \delta_{2}\right)=1$. In 1969, Baker and Davenport [1] used the theory of linear forms in logarithms of algebraic numbers to solve equations (1.1) in the particular instance $\left(a, b, c, d, \delta_{1}, \delta_{2}\right)=(1,3,8,1,-2,7)$. Since then, many systems of simultaneous Pell equations have been studied.

Many authors have obtained upper bounds for the number of solutions of (1.1) (see for example [2], [21], [22], [3], [7]). In 1996, Ono [17] remarked that the existence of only trivial solutions of the system of simultaneous Pellian equations

$$
x^{2}-a y^{2}=z^{2}-b y^{2}=1
$$

is a consequence of the related elliptic curve

$$
y^{2}=x(x+a)(x+b)
$$

having Mordell-Weil rank zero over $\mathbb{Q}$. Two years later, Bennett [2] proved that the system of simultaneous Pell equations (1.2) has at most three positive integer solutions, where $a, b$ are two distinct positive integers. In 2002, Yuan [21] strengthened this result by proving that these equations have at most two solutions in positive integers $(x, y, z)$ if $\max \{a, b\}>1.4 \cdot 10^{57}$. This result was sharpened by Bennett-Cipu-Mignotte-Okazaki [3] by removing the above condition.

Progress has been made in the study of some particular cases giving at most one positive solution (see [10], [20], [6], [23], [12], [4], [19], [11] and [14]). Moreover, very recently, Li, Xia, and Yuan [13] studied a special case

2000 Mathematics Subject Classification: 11D09, 11D25, 11B39.

Key words and phrases: quadratic and bilinear equations, simultaneous Pellian equations, Lehmer numbers. 
of system of simultaneous Pellian equations

$$
\left\{\begin{array}{l}
(m+\delta) x^{2}-m y^{2}=\delta \\
y^{2}-b z^{2}=1
\end{array}\right.
$$

where $\delta=1$ or 4 , and $2 \nmid m$ if $\delta=4$. They proved that equations (1.3) then have at most one solution in positive integers $(x, y, z)$.

In this paper, we consider an extension of the above problem. In fact, we study the system of simultaneous Pellian equations

$$
(m+\delta) x^{2}-m y^{2}=\delta, \quad y^{2}-b z^{2}=1, \quad \delta \in\{ \pm 1, \pm 2, \pm 4\},
$$

where $\min \{m, m+\delta\} \geq 1$, and $2 \nmid m$ if $\delta \neq \pm 1$. Our main result is the following.

THEOREM 1.1. Equations (1.4) have at most one solution in positive integers $(x, y, z)$.

From Theorem 1.1, we get the following result.

TheOREM 1.2. For any positive integer $m$ and $\delta \in\{ \pm 1, \pm 2, \pm 4\}$, the system of simultaneous Pellian equations

$$
x^{2}-m d z^{2}=y^{2}-(m+\delta) d z^{2}=1
$$

has at most one positive integer solution $(x, y, z)$.

In fact, if we multiply the second equation of (1.4) by $m$ and add the resulting equation to the first equation of (1.4), we obtain

$$
(m+\delta) x^{2}-m b z^{2}=m+\delta .
$$

Taking $b=d(m+\delta)$ and simplifying by $m+\delta$, we obtain (1.5). Moreover, when $d$ has the form $2^{f} p^{g}$ where $p$ is an odd prime, we can deduce that equations (1.5) have at most one positive solution $(x, y, z)$. Theorem 1.2 generalizes a theorem of Walsh [19] on equations (1.2). He proved this result for the special case $m=1$ and $\delta=1$.

The organization of this paper is as follows. In Section 2, we recall or prove some useful results following the work independently done by Yuan and Walsh. The proof of Theorem 1.1 is given in Section 3 by applying a result due to Walsh [20]. Finally, in Section 4, we study a particular case. In fact, we assume $b=b^{\prime}|4 m / \delta+4|$ with $b^{\prime} \in\{1,2, p\}$ where $p$ is an odd prime and we determine all solutions to equations (1.4).

2. Some lemmas. Let $n=\min \{m, m+\delta\}$, i.e.

$$
n= \begin{cases}m & \text { if } \delta=1,2,4, \\ m+\delta & \text { if } \delta=-1,-2,-4,\end{cases}
$$

where $2 \nmid m$ if $\delta \neq \pm 1$. Then equations (1.4) can be rewritten as

$$
\begin{gathered}
(n+c) x^{2}-n y^{2}=c, \quad c \in\{1,2,4\}, \\
y^{2}-b z^{2}=1(\text { if } \delta=c) \text { or } \quad x^{2}-b z^{2}=1(\text { if } \delta=-c) .
\end{gathered}
$$


In fact, when $\delta=-1,-2,-4$, one can interchange $x$ and $y$ to obtain equations (2.2) and (2.3). Therefore if $(x, y, z)$ is a solution of (2.2) and (2.3) when $\delta=1,2,4$, then $(y, x, z)$ is a solution of $(2.2)$ and $(2.3)$ when $\delta=-1,-2,-4$, and vice versa.

We consider the following result of Yuan [22].

LEMMA 2.1. Let $x_{1} \sqrt{a}+y_{1} \sqrt{b}$ be the smallest solution of $a x^{2}-b y^{2}=\delta$, with $\delta \in\{1,2,4\}$. Then every positive integer solution $(x, y)$ of this equation can be given by

$$
\frac{x \sqrt{a}+y \sqrt{b}}{\sqrt{\delta}}=\left(\frac{x_{1} \sqrt{a}+y_{1} \sqrt{b}}{\sqrt{\delta}}\right)^{n}, \quad n>0,
$$

with $2 \nmid n$ if $\min \{a, b\}>1$ or if $(a, \delta) \neq(1,1),(1,4)$.

We put $N=4 n / c$. Then equation (2.2) becomes

$$
(N+4) x^{2}-N y^{2}=4 .
$$

Let us consider

$$
\alpha=\frac{\sqrt{N+4}+\sqrt{N}}{2}, \quad \bar{\alpha}=\frac{\sqrt{N+4}-\sqrt{N}}{2} .
$$

By Lemma 2.1, every positive integer solution $(x, y)$ of $(2.2)$ or $(2.5)$ can be represented as

$$
\frac{x \sqrt{N+4}+y \sqrt{N}}{2}=\alpha^{n}, \quad n>0,2 \nmid n .
$$

Moreover, let $\beta=\alpha^{2}$. Then $\beta$ is the smallest solution of the equation

$$
\tau^{2} x^{2}-N(N+4) y^{2}=4
$$

where $\tau=1$ or 2 when $N$ is odd or even respectively.

Now let $\gamma=v_{1}+u_{1} \sqrt{b}$ be the smallest solution of the equation $v^{2}-b u^{2}=1$. For integers $j, k, l \geq 1$ with $2 \nmid j$, we define the sequences $\left\{T_{j}\right\},\left\{W_{j}\right\},\left\{V_{k}\right\}$, $\left\{U_{k}\right\},\left\{v_{l}\right\}$ and $\left\{u_{l}\right\}$ by

$$
\begin{aligned}
\alpha^{j} & =\frac{T_{j} \sqrt{N+4}+W_{j} \sqrt{N}}{2}, \\
\beta^{k} & =\frac{V_{k}+U_{k} \sqrt{N(N+4)}}{2}, \\
\gamma^{l} & =v_{l}+u_{l} \sqrt{b} .
\end{aligned}
$$

Notice that $(V, U)=(x, y)$ if $2 \nmid N$, and $(V, U)=(2 x, y)$ if $2 \mid N$.

The following lemma lists some properties of Lehmer sequences. They are true not only for $\left(\left\{V_{k}\right\},\left\{U_{k}\right\}\right)$, but also for $\left(\left\{T_{j}\right\},\left\{W_{j}\right\}\right)$ and $\left(\left\{v_{l}\right\},\left\{u_{l}\right\}\right)$.

LemMa 2.2. Let $d=\operatorname{gcd}(m, n)$ for some integers $m$ and $n$.

(1) If $U_{m} \neq 1$, then $U_{m} \mid U_{n}$ if and only if $m \mid n$.

(2) If $m>1$, then $V_{m} \mid V_{n}$ if and only if $n / m$ is an odd integer. 
(3) $\operatorname{gcd}\left(U_{m}, U_{n}\right)=U_{d}$.

(4) $\operatorname{gcd}\left(V_{m}, V_{n}\right)=V_{d}$ if $m / d$ and $n / d$ are odd, and 1 otherwise.

(5) $\operatorname{gcd}\left(U_{m}, V_{n}\right)=V_{d}$ if $m / d$ is even, and 1 otherwise.

Proof. See Lemma 2.1 of [23], [20], or Lemma 2.2 of [13].

One can see that if we extend the above sequences to negative indices, the definition is still effective. In fact, we have

$T_{-n}=T_{n}, W_{-n}=-W_{n}, V_{-n}=V_{n}, U_{-n}=-U_{n}, v_{-n}=v_{n}, u_{-n}=-u_{n}$.

Lemma 2.3. Let $k_{0}, k_{1}, k_{2}, p \in \mathbb{Z}$ with $k_{1}, k_{2}, p>0$. If $k_{i}(i=0,1,2)$ are all odd and $k_{2}=2 p k_{1}+k_{0}$, then

(i) $T_{2 p k_{1}+k_{0}} \equiv(-1)^{p} T_{k_{0}}\left(\bmod T_{k_{1}}\right)$;

(ii) $W_{2 p k_{1}+k_{0}} \equiv W_{k_{0}}\left(\bmod W_{k_{1}}\right)$.

Proof. (i) If $2 \nmid p$, then

$$
\begin{aligned}
T_{2 p k_{1}+k_{0}}+T_{k_{0}} & =\frac{\alpha^{2 p k_{1}+k_{0}}+\bar{\alpha}^{2 p k_{1}+k_{0}}+\alpha^{k_{0}}+\bar{\alpha}^{k_{0}}}{\sqrt{N+4}} \\
& =\frac{\left(\alpha^{p k_{1}+k_{0}}+\bar{\alpha}^{p k_{1}+k_{0}}\right)\left(\alpha^{p k_{1}}+\bar{\alpha}^{p k_{1}}\right)}{\sqrt{N+4}}=V_{\left(p k_{1}+k_{0}\right) / 2} T_{p k_{1}} .
\end{aligned}
$$

Therefore $T_{2 p k_{1}+k_{0}} \equiv-T_{k_{0}}\left(\bmod T_{k_{1}}\right)$.

If $2 \mid p$, we have

$$
\begin{aligned}
T_{2 p k_{1}+k_{0}}-T_{k_{0}} & =\frac{\alpha^{2 p k_{1}+k_{0}}+\bar{\alpha}^{2 p k_{1}+k_{0}}-\alpha^{k_{0}}-\bar{\alpha}^{k_{0}}}{\sqrt{N+4}} \\
& =\frac{\left(\alpha^{p k_{1}+k_{0}}-\bar{\alpha}^{p k_{1}+k_{0}}\right)\left(\alpha^{p k_{1}}-\bar{\alpha}^{p k_{1}}\right)}{\sqrt{N+4}}=N W_{p k_{1}+k_{0}} U_{p k_{1} / 2} .
\end{aligned}
$$

From Lemma 2.2(1), we have $U_{k_{1}} \mid U_{p k_{1} / 2}$ and by (2.8) and (2.9), we get $U_{k_{1}}=T_{k_{1}} W_{k_{1}}$. Thus $T_{2 p k_{1}+k_{0}} \equiv T_{k_{0}}\left(\bmod T_{k_{1}}\right)$.

(ii) The proof is similar to that of (i). We have

$$
\begin{aligned}
W_{2 p k_{1}+k_{0}}-W_{k_{0}} & =\frac{\alpha^{2 p k_{1}+k_{0}}-\bar{\alpha}^{2 p k_{1}+k_{0}}-\alpha^{k_{0}}+\bar{\alpha}^{k_{0}}}{\sqrt{N}} \\
& =\frac{\left(\alpha^{p k_{1}+k_{0}}+\bar{\alpha}^{p k_{1}+k_{0}}\right)\left(\alpha^{p k_{1}}-\bar{\alpha}^{p k_{1}}\right)}{\sqrt{N}} .
\end{aligned}
$$

If $2 \nmid p$, then $W_{2 p k_{1}+k_{0}}-W_{k_{0}}=V_{\left(p k_{1}+k_{0}\right) / 2} W_{p k_{1}}$. As $W_{k_{1}} \mid W_{p k_{1}}$, we have $W_{2 p k_{1}+k_{0}} \equiv W_{k_{0}}\left(\bmod W_{k_{1}}\right)$. If $2 \mid p$, then

$$
W_{2 p k_{1}+k_{0}}-W_{k_{0}}=(N+4) T_{p k_{1}+k_{0}} U_{p k_{1} / 2} .
$$

Therefore, we get the same result.

Now we assume that positive integer solutions of (2.2) and (2.3) exist. Let $\left(x_{1}, y_{1}, z_{1}\right)$ be the positive solution with the smallest $z_{1}$, and $\left(x_{2}, y_{2}, z_{2}\right)$ 
be any other solution. Then there exist positive integers $j_{i}, l_{i}(i=1,2)$ with $2 \nmid j_{i}$ such that

$$
x_{i}=T_{j_{i}}, \quad y_{i}=W_{j_{i}}, \quad y_{i} \text { or } x_{i}=v_{l_{i}}, \quad z_{i}=u_{l_{i}} .
$$

The following result is similar to Lemma 2.4 of [23] and Lemma 2.4 of [13]. In [22], Yuan proved that for positive integers $k_{0}, k_{1}, k_{2}, p$, we have $v_{2 p k_{1} \pm k_{0}} \equiv \pm v_{k_{0}}\left(\bmod v_{k_{1}}\right)$. We use it and Lemma 2.3 to get

LEMMA 2.4. In the notations of (2.11), we have

$$
y_{1}\left|y_{2}, \quad j_{1}\right| j_{2}, \quad \text { and } \quad l_{1} \mid l_{2} \text {. }
$$

Furthermore, $j_{2} / j_{1}$ and $l_{2} / l_{1}$ are odd integers. This implies $x_{1} \mid x_{2}$ and $z_{1} \mid z_{2}$.

Define

$$
R_{2 k+1}^{(\lambda)}= \begin{cases}T_{2 k+1} & \text { if } \lambda=1, \\ W_{2 k+1} & \text { if } \lambda=-1 .\end{cases}
$$

Then from the definition of $\alpha$ and (2.8) we obtain

$$
R_{2 k+1}^{(\lambda)}=\frac{\alpha^{2 k+1}+\lambda \bar{\alpha}^{2 k+1}}{\sqrt{N+2+2 \lambda}} .
$$

Lemma 2.5. We have $\left(R_{2 k+1}^{(\lambda)}\right)^{2}-1=(N+2-2 \lambda) U_{k} U_{k+1}$.

Proof. Since $\alpha+\lambda \bar{\alpha}=\sqrt{N+2+2 \lambda}$, we get

$$
\begin{aligned}
\left(R_{2 k+1}^{(\lambda)}\right)^{2}-1 & =\left(\frac{\alpha^{2 k+1}+\lambda \bar{\alpha}^{2 k+1}}{\sqrt{N+2+2 \lambda}}\right)^{2}-1=\frac{\alpha^{4 k+2}+\bar{\alpha}^{4 k+2}+2 \lambda}{N+2+2 \lambda}-1 \\
& =\frac{\alpha^{4 k+2}+\bar{\alpha}^{4 k+2}-(N+2)}{N+2+2 \lambda}=\frac{\beta^{2 k+1}+\bar{\beta}^{2 k+1}-(\beta+\bar{\beta})}{N+2+2 \lambda} \\
& =\frac{\left(\beta^{k+1}-\bar{\beta}^{k+1}\right)\left(\beta^{k}-\bar{\beta}^{k}\right)}{N+2+2 \lambda} \\
& =\frac{N(N+4)}{N+2+2 \lambda} \cdot \frac{\beta^{k}-\bar{\beta}^{k}}{\sqrt{N(N+4)}} \cdot \frac{\beta^{k+1}-\bar{\beta}^{k+1}}{\sqrt{N(N+4)}} \\
& =(N+2-2 \lambda) U_{k} U_{k+1} .
\end{aligned}
$$

Definition 2.6. Let $\left\{U_{k}\right\}$ be defined by (2.9). If there is a prime factor $p$ of $U_{k}$ that does not divide $U_{j}$ for all $1 \leq j \leq k-1$, then we say that $p$ is a primitive prime factor of $U_{k}$.

Notice that there are two (slightly) different definitions of primitive prime factor. According to the definition in [5], $p$ should not divide $N(N+4)$ and $U_{j}$ for all $1 \leq j \leq k-1$. This was used in [23] and [13]. But the above definition is enough for our proof. 
Lemma 2.7. For $k>1, U_{k}$ has a primitive prime factor $p$ except for $\beta=(1+\sqrt{5}) / 2$ and $k=6$. Moreover, $p \mid U_{k^{\prime}}$ if and only if $k \mid k^{\prime}$.

Proof. See Lemma 2.4 of [20].

The following result is an adaptation of Lemma 2.5 of [20]. One can get it from some results on $A X^{2}-B y^{4}=1,4$ due to Ljunggren [15], Cohn [8], [9] and the first author [18].

Lemma 2.8. Let $\tau=1$ if $2 \nmid N$, and $\tau=2$ if $2 \mid N$. Then for any positive integer $A$, there is at most one positive solution $(x, y)$ to the equation

$$
\tau^{2} x^{2}-N(N+4) y^{2}=4
$$

with $y=A u^{2}$ for some integer $u$, except in the following cases:

(1) $N=1, A=1$, in which case $y \in\left\{1,12^{2}\right\}$.

(2) $N=336, A=1$, in which case $y \in\left\{1,6214^{2}\right\}$.

(3) $N=d^{2}-2, A=1$, in which case $y \in\left\{1, d^{2}\right\}$.

Proof. Take $M=N+2, X=\tau x$, and $Y=y$ in Lemma 2.5 of [20]. Moreover, if $N$ is even, one can take $N=2 M-2$, and if $N$ is odd, $N=$ $M-2$.

Next, we recall the following result due to Ljunggren [16].

Lemma 2.9. The Diophantine equation

$$
x^{4}-p y^{2}=1,
$$

where $p$ denotes any odd prime, has no solutions in positive integers $x$ and $y$ if $p \neq 5$ and $p \neq 29$. When $p=5$ or $p=29$ there is only one solution, i.e. $(x, y)=(3,4)$ and $(x, y)=(99,1820)$ respectively.

3. Proof of Theorem 1.1. In this section, we will prove the main theorem of this paper. We assume that $\left(x_{1}, y_{1}, z_{1}\right)$ is the positive solution with the smallest positive $z_{1}$, and $\left(x_{2}, y_{2}, z_{2}\right)$ is any other positive solution of equations (2.2) and (2.3). Then there exist positive integers $j_{i}, l_{i}(i=1,2)$ with $2 \nmid j_{i}$ such that

$$
x_{i}=T_{j_{i}}, \quad y_{i}=W_{j_{i}}, \quad y_{i} \text { or } x_{i}=v_{l_{i}}, \quad z_{i}=u_{l_{i}} .
$$

We notice that $j_{1}>1$, otherwise $T_{1}=W_{1}=1$. This implies $l_{1}=1, v_{l_{1}}=1$ and $z=0$. Let $j_{i}=2 k_{i}+1(i=1,2)$ with $0<k_{1}<k_{2}$. From (2.3) and (3.1), we have $T_{2 k_{i}+1}^{2}-1=b z_{i}^{2}$ or $W_{2 k_{i}+1}^{2}-1=b z_{i}^{2}$. Using (2.12) and Lemma 2.5, we get

$$
\begin{gathered}
b z_{1}^{2}=\left(R_{2 k_{1}+1}^{(\lambda)}\right)^{2}-1=(N+2-2 \lambda) U_{k_{1}} U_{k_{1}+1}, \\
b z_{2}^{2}=\left(R_{2 k_{2}+1}^{(\lambda)}\right)^{2}-1=(N+2-2 \lambda) U_{k_{2}} U_{k_{2}+1} .
\end{gathered}
$$


From Lemma 2.4, we have $z_{1} \mid z_{2}$, so (3.2) and (3.3) give

$$
\frac{U_{k_{2}} U_{k_{2}+1}}{U_{k_{1}} U_{k_{1}+1}}=\left(\frac{z_{2}}{z_{1}}\right)^{2} \text {. }
$$

Before discussing the above equation, let us express $U_{k}(1 \leq k \leq 6)$ using the recurrence relation $U_{k+2}=(N+2) U_{k+1}-U_{k}$ for $k \geq 1$ :

$$
\begin{aligned}
& U_{1}=1, \\
& U_{2}=N+2, \\
& U_{3}=N^{2}+4 N+3, \\
& U_{4}=N^{3}+6 N^{2}+10 N+4, \\
& U_{5}=N^{4}+8 N^{3}+21 N^{2}+20 N+5, \\
& U_{6}=N^{5}+10 N^{4}+36 N^{3}+56 N^{2}+35 N+6 .
\end{aligned}
$$

First, we assume that $N=1, k_{1}=5$ or 6 . If $k_{1}=5$, then $U_{k_{1}+1}=144=$ $2^{4} \cdot 3^{2}$. By Lemma 2.7, $U_{k_{1}}$ has a primitive prime factor $p$, so that $U_{k_{1}} \mid U_{k_{2}}$ or $U_{k_{1}} \mid U_{k_{2}+1}$. If $U_{k_{1}} \mid U_{k_{2}}$, since $\operatorname{gcd}\left(U_{k_{2}}, U_{k_{2}+1}\right)=1$, equation (3.4) implies the existence of positive integers $s$ and $t$ such that $U_{k_{2}} /\left(144 U_{k_{1}}\right)=s^{2}$, $U_{k_{2}+1}=t^{2}$ or $U_{k_{2}} /\left(16 U_{k_{1}}\right)=s^{2}, U_{k_{2}+1} / 9=t^{2}$ or $U_{k_{2}} /\left(9 U_{k_{1}}\right)=s^{2}$, $U_{k_{2}+1} / 16=t^{2}$ or $U_{k_{2}} / U_{k_{1}}=s^{2}, U_{k_{2}+1} / 144=t^{2}$. The above cases give us $U_{k_{2}+1}=\square$. Using Lemma 2.8 and $U_{1}=1$, one can see that $U_{k_{2}+1}=144$ and $k_{2}=5$. This contradicts the fact that $k_{1}<k_{2}$. If $U_{k_{1}} \mid U_{k_{2}+1}$, then $k_{2}=6$. This is impossible. In the same way, if $k_{1}=6$, we also get a contradiction.

Assume now $N>1$ or $N=1, k_{1} \neq 5,6$. If $k_{1}>1$, by Lemma 2.7, $U_{k_{1}}$ and $U_{k_{1}+1}$ have primitive prime factors $p$ and $q$ respectively. By Lemma 2.7 again, equation (3.4) implies that

$$
\left(k_{1} \mid k_{2} \text { or } k_{1} \mid k_{2}+1\right) \text { and }\left(k_{1}+1 \mid k_{2} \text { or } k_{2} \mid k_{2}+1\right) \text {. }
$$

If $k_{1}=1$, then $U_{2}$ has primitive prime factor $q$, and properties (3.5) also hold. Moreover, since $j_{1} \mid j_{2}$, we have

$$
2 k_{1}+1 \mid 2 k_{2}+1
$$

Note that $\operatorname{gcd}\left(U_{k_{2}}, U_{k_{2}+1}\right)=1$ by Lemma 2.2(3). Then properties (3.4) and (3.5) give us the following four cases:

$$
\begin{array}{ll}
\text { (i) } U_{k_{2}+1} /\left(U_{k_{1}} U_{k_{1}+1}\right)=s^{2}, & U_{k_{2}}=t^{2}, \\
\text { (ii) } U_{k_{2}} /\left(U_{k_{1}} U_{k_{1}+1}\right)=s^{2}, & U_{k_{2}+1}=t^{2}, \\
\text { (iii) } U_{k_{2}} / U_{k_{1}+1}=s^{2}, & U_{k_{2}+1} / U_{k_{1}}=t^{2}, \\
\text { (iv) } U_{k_{2}} / U_{k_{1}}=s^{2}, & U_{k_{2}+1} / U_{k_{1}+1}=t^{2} .
\end{array}
$$

CASe (i). Since $(V, U)=(N+2,1)$ is a solution of $V^{2}-N(N+4) U^{2}=4$, using equations (3.7) one can see that the equations 


$$
\tau^{2} x^{2}-N(N+4) y^{2}=4, \quad y=u^{2}
$$

have two solutions $u=1$ and $u=t>1$. By Lemma 2.8, we obtain $N=1$, 336 , or $d^{2}-2$.

- If $N=1$, then we get $t=12$. Therefore, equations (3.11) imply $x^{2}-5 y^{2}=4$. Any solution $(x, y)$ is given by

$$
\frac{x+y \sqrt{5}}{2}=\left(\frac{3+\sqrt{5}}{2}\right)^{k} .
$$

The solution with $y=144$ implies $k_{2}=6$. On the other hand, the first equation of (3.7) gives us $k_{1}\left(k_{1}+1\right) \mid k_{2}+1=7$. This is impossible.

- If $N=336$, then $t=6214$. From $U_{4}=N^{3}+6 N^{2}+10 N+4=6214^{2}$, we obtain $k_{2}=4$. Since $k_{1}\left(k_{1}+1\right) \mid k_{2}+1=5$, we can find no positive integer $k_{1}$.

- If $N=d^{2}-2$, then $t=d$. From $U_{2}=N+2=t^{2}$, we get $k_{2}=2$. But there is no positive integer $k_{1}$ satisfying $k_{1}\left(k_{1}+1\right) \mid k_{2}+1=3$.

CASe (ii). This is similar to Case (i). By Lemma 2.8, the second equation of (3.8) implies $N=1,336$, or $d^{2}-2$.

- If $N=1$, then $k_{2}+1=6$. We have already discussed this case and it is impossible.

- If $N=336$, then $k_{2}+1=4$. But $k_{1}\left(k_{1}+1\right) \mid k_{2}=3$ is also impossible.

- If $N=d^{2}-2$, then $k_{2}+1=2$. But there is no integer $k_{1}$ such that $0<k_{1}<k_{2}$.

CASE (iii). From (3.9), we have

$$
U_{k_{1}+1}=A s_{1}^{2}, \quad U_{k_{2}}=A s_{2}^{2}, \quad U_{k_{1}}=B t_{1}^{2}, \quad U_{k_{2}+1}=B t_{2}^{2}
$$

for some positive integers $A, B, s_{1}, s_{2}, t_{1}, t_{2}$ such that $s=s_{2} / s_{1}$ and $t=t_{2} / t_{1}$. If $k_{1}+1=k_{2}$, from (3.6) we get $2 k_{1}+1 \mid 2 k_{1}+3$, which is impossible. Therefore we consider $k_{1}+1<k_{2}$. Thus $U_{k_{1}}<U_{k_{1}+1}<U_{k_{2}}<U_{k_{2}+1}$. But from Lemma $2.8, A=B=1$ and $N=1,336$ or $d^{2}-2$. Then $U_{k_{1}}, U_{k_{1}+1}, U_{k_{2}}$, and $U_{k_{2}+1}$ are all perfect squares. This leads to a contradiction.

Case (iv). From (3.10), as in Case (iii), we have

$$
U_{k_{1}}=A s_{1}^{2}, \quad U_{k_{2}}=A s_{2}^{2}, \quad U_{k_{1}+1}=B t_{1}^{2}, \quad U_{k_{2}+1}=B t_{2}^{2} .
$$

Since $k_{1}<k_{2}$, from Lemma 2.8 we have $A=B=1$ and $N=1,336$, or $d^{2}-2$. We get a contradiction as before. This completes the proof of Theorem 1.1.

4. A particular case. Now we consider equations (1.4) with

$$
b=b^{\prime}|4 m / \delta+4|, \quad b^{\prime} \in\{1,2, p\} .
$$

Then we have the following result. 
Proposition 4.1. If equations (1.4) have a solution $(x, y, z)$ with the condition (4.1), then $U_{k} U_{k+1}=b^{\prime} z^{2}$ when either

or

$$
N=b^{\prime} d^{2}-2, \quad b^{\prime} \in\{1,2, p\}, \quad k=1, \quad z=d ;
$$

$$
N=7, \quad b^{\prime}=5, \quad k=2, \quad z=12 ;
$$

or

$$
N=9799, \quad b^{\prime}=29, \quad k=2, \quad z=180180 .
$$

Proof. Suppose a positive integer solution $(x, y, z)$ of $(1.4)$ exists. Then there are positive integers $j, l$ with $2 \nmid j$ such that

$$
x=T_{j}, \quad y=W_{j}, \quad y \text { or } x=v_{l}, \quad z=u_{l} .
$$

If $j=1$ then $z=0$. Let $j=2 k+1$ for $k>0$. From (2.3) and (4.2) we obtain $T_{2 k+1}^{2}-1=b z^{2}$ or $W_{2 k+1}^{2}-1=b z^{2}$. Using (2.12) and Lemma 2.5, we get $b z^{2}=\left(R_{2 k+1}^{(\lambda)}\right)^{2}-1=(N+2-2 \lambda) U_{k} U_{k+1}$. Thus

$$
(N+2-2 \lambda) U_{k} U_{k+1}=b^{\prime}|4 m / \delta+4| z^{2}, \quad b^{\prime} \in\{1,2, p\} .
$$

We recall that $N=4 n / c, n=\min \{m, m+\delta\}$ and $c=|\delta|$.

If $\delta \in\{1,2,4\}$, then equations (1.4) give us the first equation in (2.3) and $n=m, c=\delta$. Thus we need to consider $W_{2 k+1}^{2}-1=b z^{2}$. By the definition of $R_{2 k+1}^{(\lambda)}$ in (2.12), we have $\lambda=-1$. Therefore one can see that

$$
N+2-2 \lambda=4 m / c+4=|4 m / \delta+4| .
$$

If $\delta \in\{-1,-2,-4\}$, then equations (1.4) give us the second equation in $(2.3)$ and $n=m-c, c=-\delta$. Thus we need to consider $T_{2 k+1}^{2}-1=b z^{2}$ and $\lambda=1$. Therefore we also obtain

$$
N+2-2 \lambda=4(m-c) / c=4 m / c-4=|4 m / \delta+4| .
$$

Then equation (4.3) implies

$$
U_{k} U_{k+1}=b^{\prime} z^{2}, \quad b^{\prime} \in\{1,2, p\} .
$$

By Lemma 2.2(3), we have $\operatorname{gcd}\left(U_{k}, U_{k+1}\right)=1$. So we obtain either

$$
U_{k}=s^{2}, \quad U_{k+1}=b^{\prime} t^{2},
$$

or

$$
U_{k}=b^{\prime} t^{2}, \quad U_{k+1}=s^{2},
$$

where $z=s t, s, t \in \mathbb{N}$.

If equations (4.5) hold, then from Lemma 2.8 one can see that $U_{k}=s^{2}$ implies $k=1$, except for $N=1,336$, or $d^{2}-2$. First, we suppose $k=1$. Then from the second equation of (4.5) we have $U_{2}=N+2=b^{\prime} t^{2}$. Thus $N=b^{\prime} t^{2}-2$ with $b^{\prime} \in\{1,2, p\}$. Second, we suppose $k>1$ and we discuss the following three cases. 
- If $N=1$, then $U_{k}$ is a perfect square when $k=6$. But $b^{\prime} t^{2}=U_{k+1}=$ $U_{7}=(N+2) U_{6}-U_{5}=377=13 \cdot 29$ is impossible.

- If $N=336$, then $k=4$. The fact that $b^{\prime} t^{2}=U_{k+1}=U_{5}=N^{4}+8 N^{3}+$ $21 N^{2}+20 N+5=13051348805=5 \cdot 11 \cdot 19 \cdot 109 \cdot 149 \cdot 769$ also leads to a contradiction.

- If $N=d^{2}-2$, then $k=2$ and $s=d$. Therefore, from $b^{\prime} t^{2}=U_{k+1}=$ $U_{3}=N^{2}+4 N+3=(N+2)^{2}-1$, we have

$$
d^{4}-b^{\prime} t^{2}=1, \quad b^{\prime} \in\{1,2, p\} .
$$

It is easy to see that (4.7) has no positive integer solution when $b^{\prime}=1$. If $b^{\prime}=2$, then (4.7) implies $d=1, t=0$, which is impossible. If $b^{\prime}=p$, then by Lemma 2.9, equation (4.7) has a positive integer solution if and only if either $b^{\prime}=5,(d, t)=(3,4)$, or $b^{\prime}=29,(d, t)=(99,1820)$. Since $z=s t$, we get $z=12$ or 180180 respectively.

Now we suppose equations (4.6) hold. In a similar way, $U_{k+1}=s^{2}$ implies $k=0$, except for $N=1,336$, or $d^{2}-2$. But $k=0$ leads to a contradiction. Now we discuss the following three cases when $k>0$.

- If $N=1$, then $k+1=6$. But $b^{\prime} t^{2}=U_{k}=U_{5}=N^{4}+8 N^{3}+21 N^{2}+$ $20 N+5=55=5 \cdot 11$ gives a contradiction.

- If $N=336$, then $k+1=4$. Thus $b^{\prime} t^{2}=U_{k}=U_{3}=N^{2}+4 N+3=$ $114243=3 \cdot 113 \cdot 337$ is impossible.

- If $N=d^{2}-2$, then $k+1=2$. Then from $b^{\prime} t^{2}=U_{1}=1$, we get $b^{\prime}=1$ and $t=1$. This is also impossible.

Finally, we use Proposition 4.1 to prove the following result which is a particular case of Theorem 1.1.

Theorem 4.2. If $p$ is an odd prime and $b=b^{\prime}|4 m / \delta+4|, b^{\prime} \in\{1,2, p\}$, then the system of simultaneous equations (1.4) has no positive integer solution $(x, y, z)$, except in the following cases.

(1) If $\left(\delta, b^{\prime}\right) \neq( \pm 1,1),( \pm 1, p)$, then there is a positive integer $d$ such that

$$
m= \begin{cases}\delta\left(b^{\prime} d^{2}-2\right) / 4 & \text { if } \delta>0, \\ -\delta\left(b^{\prime} d^{2}+2\right) / 4 & \text { if } \delta<0,\end{cases}
$$

and equations (1.4) have one solution

$$
(x, y, z)=(|4 m / \delta+1|,|4 m / \delta+3|, d) .
$$

(2) If $(m, \delta, b)=(7,4,55)$, then the solution is $(x, y, z)=(71,89,12)$; if $(m, \delta, b)=(11,-4,35)$, then the solution is $(x, y, z)=(89,71,12)$.

(3) If $(m, \delta, b)=(9799,4,29 \cdot 9803)$, then the solution is

$$
(x, y, z)=(96049799,96069401,180180) \text {; }
$$




$$
\text { if } \begin{aligned}
(m, \delta, b)= & (9803,-4,29 \cdot 9799), \text { then the solution is } \\
& (x, y, z)=(96069401,96049799,180180) .
\end{aligned}
$$

Proof. Suppose that there exists a positive integer solution $(x, y, z)$ of equations (1.4) with the condition (4.1). From Proposition 4.1, we have $U_{k} U_{k+1}=b^{\prime} z^{2}, b^{\prime} \in\{1,2, p\}$ and $N=4 n /|\delta|=b^{\prime} d^{2}-2,7$ or 9799 , where $n=\min \{m, m+\delta\}$.

First, let $N=b^{\prime} d^{2}-2$. If $\delta>0$, then $n=m$, thus $m=\delta\left(b^{\prime} d^{2}-2\right) / 4$. We have $k=1$ and $z=d$ by Proposition 4.1. From $y^{2}=b z^{2}+1$ we obtain

$$
\begin{aligned}
y^{2} & =b^{\prime}|4 m / \delta+4| z^{2}+1=b^{\prime}(4 m / \delta+4) z^{2}+1 \\
& =b^{\prime}\left(b^{\prime} d^{2}+2\right) d^{2}+1=\left(b^{\prime} d^{2}+1\right)^{2} .
\end{aligned}
$$

Thus we have $y=b^{\prime} d^{2}+1=4 m / \delta+3$. Consequently, we get the solution $(x, y, z)=(4 m / \delta+1,4 m / \delta+3, d)$.

If $\delta<0$, then $n=m+\delta$, thus $m=-\delta\left(b^{\prime} d^{2}-2\right) / 4-\delta=-\delta\left(b^{\prime} d^{2}+2\right) / 4$. In a similar way, knowing that $\delta+m \geq 1$ we have

$$
\begin{aligned}
y^{2} & =b^{\prime}|4 m / \delta+4| z^{2}+1=b^{\prime}(4 m /(-\delta)-4) z^{2}+1 \\
& =b^{\prime}\left(b^{\prime} d^{2}-2\right) d^{2}+1=\left(b^{\prime} d^{2}-1\right)^{2} .
\end{aligned}
$$

It follows that $y=b^{\prime} d^{2}-1=4 m /(-\delta)-3$, and we get the solution $(x, y, z)=$ $(4 m /(-\delta)-1,4 m /(-\delta)-3, d)$. This proves the first exceptional case.

Finally, let $N=7$ or 9799 . Since $N=4 n /|\delta|$, we have $|\delta|=4$. Noticing $k=2$, by direct computations, it is easy to get the second and third exceptional cases. This completes the proof of Theorem 4.2.

Acknowledgments. We thank Prof. Yuan for informing us about the paper [14] and for sending us a copy. Li and Yuan have studied the same system and obtained a result similar to our Theorem 1.1 but using another method.

The authors thank Gary Walsh for many helpful suggestions and comments during the preparation of this paper, particularly for those leading to Theorem 1.2. The authors express their gratitude to the anonymous referee for constructive suggestions to improve an earlier draft of this paper. The first author is partially supported by Purdue University North Central. The second author is supported by the Natural Science Foundation of Education Department of Sichuan Province (No. 2006C057).

\section{References}

[1] A. Baker and H. Davenport, The equations $3 x^{2}-2=y^{2}$ and $8 x^{2}-7=z^{2}$, Quart. J. Math. Oxford Ser. (2) 20 (1969), 129-137.

[2] M. A. Bennett, On the number of solutions of simultaneous Pell equations, J. Reine Angew. Math. 498 (1998), 173-199. 
[3] M. A. Bennett, M. Cipu, M. Mignotte and R. Okazaki, On the number of solutions of simultaneous Pell equations II, Acta Arith. 122 (2006), 407-417.

[4] M. A. Bennett and P. G. Walsh, Simultaneous quadratic equations with few or no solutions, Indag. Math. 11 (2000), 1-12.

[5] Yu. Bilu, G. Hanrot and P. M. Voutier (with an appendix by M. Mignotte), Existence of primitive divisors of Lucas and Lehmer numbers, J. Reine Angew. Math. 539 (2001), 75-122.

[6] M. Cipu, Pairs of Pell equations having at most one common solution in positive integers, An. Ştiinţ. Univ. "Ovidius" Constanţa 15 (2007), 55-66.

[7] M. Cipu and M. Mignotte, On the number of solutions of simultaneous hyperbolic Diophantine equations, J. Number Theory 125 (2007), 356-392.

[8] J. H. E. Cohn, Eight Diophantine equations, Proc. London Math. Soc. 16 (1966), 153-166.

[9] —, Five Diophantine equations, Math. Scand. 21 (1967), 61-70.

[10] X. L. Dong, W. C. Shiu, C. I. Chu and Z. F. Cao, The simultaneous Pell equations $y^{2}-D z^{2}=1$ and $x^{2}-2 D z^{2}=1$, Acta Arith. 126 (2007), 115-123.

[11] B. He, On the number of solutions of simultaneous Pell equations $x^{2}-a y^{2}=1$ and $y^{2}-b z^{2}=1$, Acta Math. Sinica 51 (2008), 721-726 (in Chinese).

[12] S.-I. Katayama and C. Levesque, On simultaneous diophantine equations, Acta Arith. 108 (2003), 369-377.

[13] Z. G. Li, J. Y. Xia and P. Z. Yuan, On some special forms of simultaneous Pell equations, ibid. 128 (2007), 55-67.

[14] Z. G. Li and P. Z. Yuan, On the number of solutions of some special simultaneous Pell equations, Acta Math. Sinica 50 (2007) 1349-1356 (in Chinese).

[15] W. Ljunggren, Einige Eigenschaften der Einheiten reeller quadratischer und reinbiquadratischer Zahlkörper mit Anwendung auf die Lösung einer Klasse unbestimmter Gleichungen vierten Grades, Oslo Vid.-Akad. Skrifter 1936, no. 12, 1-73.

[16] - Some remarks on the diophantine equations $x^{2}-d y^{4}=1$ and $x^{4}-d y^{2}=1$, J. London Math. Soc. 41 (1966), 542-544.

[17] K. Ono, Euler's concordant forms, Acta Arith. 78 (1996), 101-123.

[18] A. Togbé, P. M. Voutier and P. G. Walsh, Solving a family of Thue equations with an application to the equation $x^{2}-D y^{4}=1$, ibid. 120 (2005), 39-58.

[19] P. G. Walsh, On integer solutions to $x^{2}-d y^{2}=1, z^{2}-2 d y^{2}=1$, ibid. 82 (1997), 69-76.

[20] —, Sharp bounds for the number of solutions to simultaneous Pellian equations, ibid. 126 (2007), 125-137.

[21] P. Z. Yuan, On the number of solutions of simultaneous Pell equations, ibid. 101 (2002), 215-221.

[22] -, Simultaneous Pell equations, ibid. 115 (2004), 119-131.

[23] - On the number of solutions of $x^{2}-4 m(m+1) y^{2}=y^{2}-b z^{2}=1$, Proc. Amer. Math. Soc. 132 (2004), 1561-1566.

Mathematics Department

Purdue University North Central

1401 S, U.S. 421

Westville, IN 46391, U.S.A.

E-mail: atogbe@pnc.edu
Key Laboratory of Numerical Simulation of Sichuan Province

Neijiang Normal University Neijiang, Sichuan, 641112, P.R. China E-mail: hebo-one@hotmail.com 